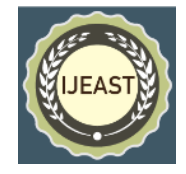

\title{
IN VEHICLE INFOTAINMENT SYSTEM USING HAND GESTURES
}

\author{
Megha $S$ \\ Department of CNE \\ Dr. Ambedkar Institute of technology, \\ Blore, India
}

\author{
Pushpalatha. S \\ Department of CNE \\ Dr. Ambedkar Institute of technology, \\ Blore, India
}

\begin{abstract}
In Vehicle Infotainment (IVI) is term that refers to vehicle systems that combines both entertainment and information delivery to drivers and passengers. These systems use audio as well as video interfaces, touch screens, keypads and other types of devices to provide these types of services. The recognition of dynamic gestures from static poses happens via a simple start-end definition of the gesture. The design of this system with an intuitive, robust, human machine interface based on the hand gestures which is been recognized by the machine. It relay's on the raspberry pi camera which recognizes the hand gestures and systematically assigned features for those actions.
\end{abstract}

Keywords: IVI, Hand gestures, Raspberry-Pi.

\section{INTRODUCTION}

The detection of objects and tracking in anyone's hands, is widely used over the computer vision communication, which has resulted. the hands are the most important for communication which is implemented in human integrated machine. Taking the information from the hand's movement is a very important activity as it may provide different information related to the vehicle like changing of the music, or driver's attention, etc. In most of the days, driver's distraction has lead to various accidents like, eyes distraction on road time and glance duration. Performing various activities has lead to many accidents, therefore instead of driver's attention put towards other activities while driving, he can just use his hand. The design of this system with an intuitive, robust, human machine interface based on the hand gestures which is been recognised by the machine. It relay's on the raspberry pi camera which recognises the hand gestures and systematically assigned features for those actions. The tracking of an object with respect to human hands is in use with the computer vision technology as the human hands are keenly important means of communication. The activity of the hands are very important in human machine interaction as they may provide information regarding driver's attentiveness. Driver's distraction may lead to various accidents, for example, most of the accidents occur due to the usage of mobile phones, while attending calls or any activities, hence its important to keep a track on driver's attention.

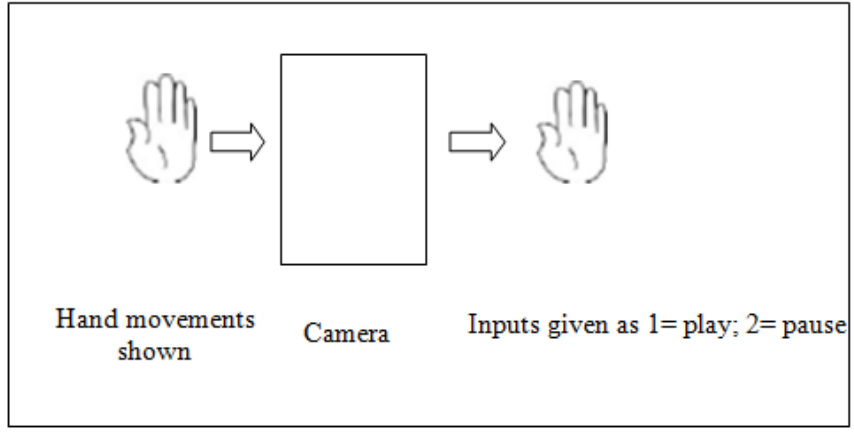

Fig 1. Actual processing of IVI

\section{REQUIREMENTS}

\section{O/S : Windows XP/7/8/10, Webcam}

2. Language : Java

\section{IDE : MATLAB.}

The specific needs of the In vehicle system includes

The Constraints restrict how functional requirements can be carried out. The In Vehicle Infotainment system can be used with the standard two way communication with the voice commands. It includes rear seat capability which allows passengers to control the visual media. Newer vehicles have range of systems which allows the devices and even the laptops to connect to the vehicle for the passenger use. . The major areas to concentrate over the designing purpose are:

1. Global technical Restrictions: For any system to operate properly, the captured image should have minimum range of disturbance, thereby the threshold will be minimum. Other than this, the camera should be small enough to ensure there is no motion other than the hand movements. The depth of the field image should ensure that the hand should focus only within the range. All these restrictions are to be fulfilled in large amount of division through finite state machine. L. Bao and S. Intille et al.(2004) describes about the importance of the hand gestures while driving.

2. Constraints of the Application Scenario:The computer Vision algorithms should be able to extract the data from the frames, to operate the infotainment service system for the clear understanding of it its 
constraints can even save thousands of code errors which makes the application work robustly, for example, given a fixed location of the camera on the ceiling.

\section{HAND GESTURE FUNCTIONS}

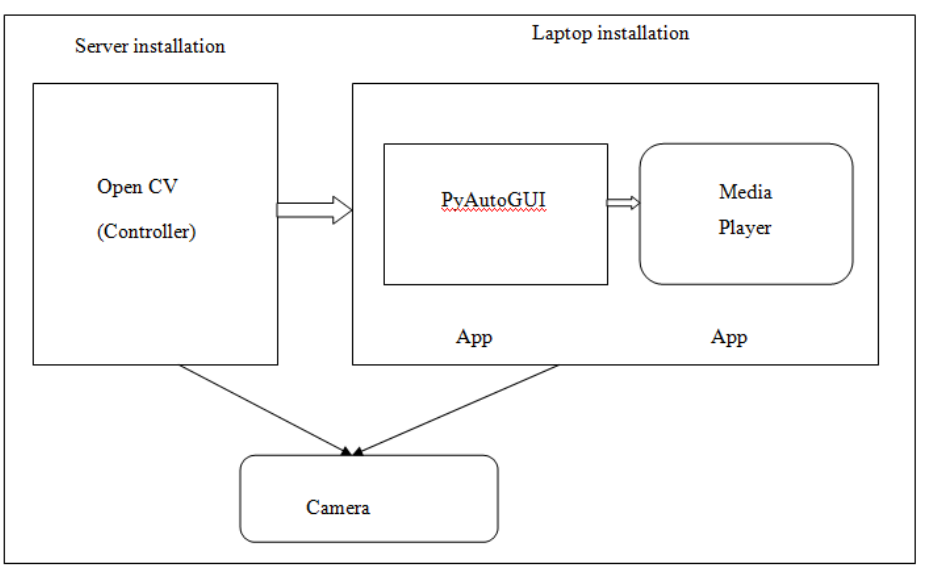

Fig 2. Design of IVI System

The hand gestures are relatable dynamic that $\mathrm{n}$ cant be changed over the state, therefore in the vehicle infotainment system, a static hand pose is all that matters. Akyol et al.(2000) emphasis on the various methods by hand mechanism in the work. In an vehicle infotainment system, the first audio channel can be done either by giving the inputs as by the numbers with using the fingers or by zooming in and zooming out of the fingers. The hand movements are recognised by the camera and the inputs can be fed within the code which can be given as the output. A static hand recognition by the most frequent recognition within the series should be above threshold that is lower disturbance. the premise of process dynamic hand postures ought to be at purpose with time allowed by the occurrences.

I. Example one or two with the finger movement.

II. The next important feature is to attach web camera for the further use of the code, the initially used code can be run with or without the attachment of this camera.

III. The next step is to generate or develop a code for providing an input for the movements of the hand with the help of Java, but on a different platform, say LINUX. The code generated uses MATLAB which is the library function of python helps in linking with the media player. After both the code development, integration of both codes has to be done for the working with the media player.

IV. Compilation of both codes that is code that is generated for hand recognition on windows and other code for feed input has to be done.

V. Checking for the code, whether if the inputs feed is working with the media player as well as is it recognising the hand movements. The various actions that are fed inside the machine learning to possess the actual actions. Many In Vehicle System allows the passengers to communicate with the machine of the system. J. Mantyjarv et al.(2001) emphasis on the various movements of the hand which may provide various actions. The vehicle industry is the top adopters of the technical innovations for various purposes like enhancing engine working, security purposes and even the delivery economic services. The IVI system can include playing the audio content, video content listening to the calls while answering without the use of phones, accessing internet enabled android system for map recognition, sports update or weather forecast.

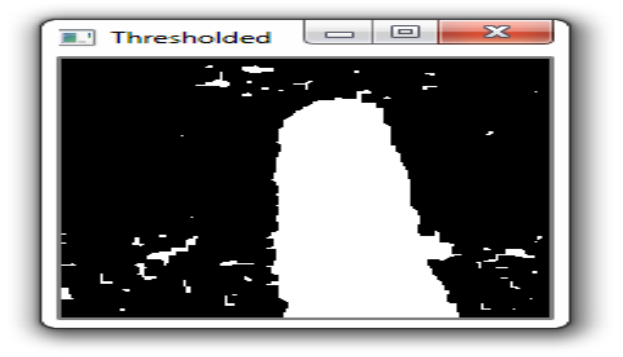

Fig 3. Hand recognition for controlling media

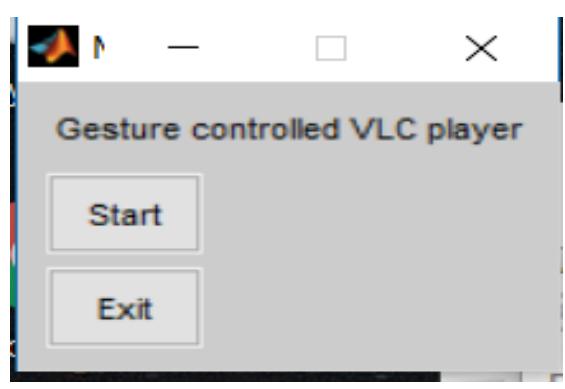

Fig 4. Gesture controlling using MATLAB

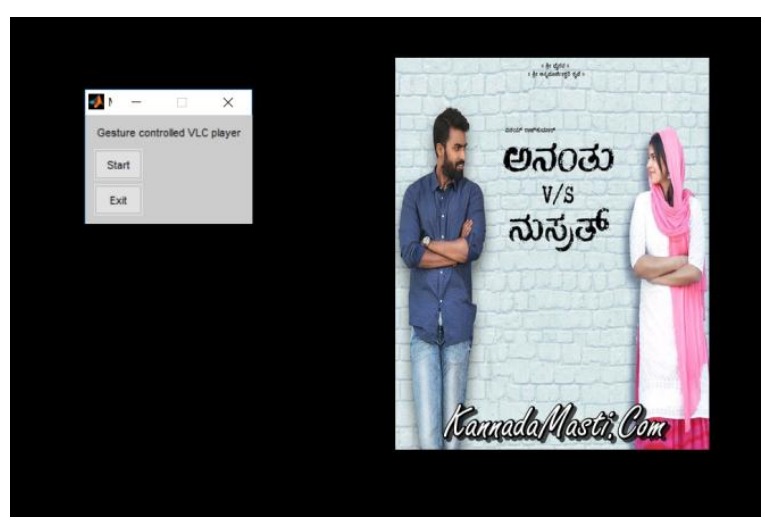

Fig 5. Media-coltrol

\section{CONCLUSION}

Performing various activities has lead to many accidents, therefore instead of driver's attention put towards other activities while driving, he can just use his hand. The design of this system with an intuitive, robust, human machine interface based on the hand gestures which can be recognised by the machine. It relay's on the raspberry pi camera which recognises 


\section{International Journal of Engineering Applied Sciences and Technology, 2019 \\ Vol. 4, Issue 3, ISSN No. 2455-2143, Pages 117-119 \\ Published Online July 2019 in IJEAST (http://www.ijeast.com)}

the hand gestures and systematically assigned features for those actions. Performing various activities has lead to many accidents, therefore instead of driver's attention put towards other activities while driving, he can just use his hand. The design of this system with an intuitive, robust, human machine interface based on the hand gestures which can be recognised by the machine. It relay's on the raspberry pi camera which recognises the hand gestures and systematically assigned features for those actions.

\section{ACKNOWLEDGEMNT}

To all the authors, from whom I have taken the help for the paper preparation. I want to thank my guide for the support she has given me in the development of the paper.

\section{REFERENCES}

[1] J. Mantyjarvi, J. Himberg, and T. Seppanen,, "Recognizing human motion with multiple acceleration sensors," in 2001 IEEE International Conference on Systems, Man and Cybernetics, Vol. 3494, 2001, pp.

[2] L. Bao and S. Intille, "Activity recognition from user-annotated acceleration data," in Pervasive Computing, F. Mattern, Ed., 2004.

[3] D. Bannach, K. Kunze, P. Lukowicz, and O. Amft, "Distributed modular toolbox for multi-modal context recognition," in Proceedings of the 19th International Conference on the Architecture of Computing Systems (ARCS 2006), LNCS, SpringerVerlag, 2006.

[4] E. Keogh, S. Chu, D. Hart, and M. Pazzani, "An online algorithm for segmenting time series," in Proceedings of the IEEE International Conference on Data Mining, 2001.

[5] L. Seon-Woo and K. Mase, "Recognition of walking behaviors for pedestrian navigation," in Proc. of the 2001 IEEE International
Conference on Control Applications (CCA'01), 2001, pp. 1152-1155.

[6] J. Matas, O. Chum, M. Urban and T. Pajdla, "Robust Wide Baseline Stereo from Maximally Stable Extremal Regions," Proc. 13th British Machine Vision Conf., pp. 384-393, 2002.

[7] Klauer, S. G., Guo, F., Sudweeks, J., \& Dingus, T. A. (2010). An analysis of driver inattention using a case-crossover approach on 100-car data: Final report” (No. HS-811 334).

[8] OpenCV Documentation. http://www.opencv.org [9] Akyol S., Canzler U., Bengler K., Hahn W. "Gesture Control for Use in Automobiles" in Proceedings of the IAPR MVA 2000 Workshop on Machine Vision Applications, pp. 349-352, November 28-30, 2000, Tokyo .

[10] Van den Bergh, M., \& Van Gool, L. "Combining RGB and ToF cameras for real-time 3D hand gesture interaction." In Applications of Computer Vision (WACV), 2011 IEEE Workshop on. pp. 66-72. January 2011.

[11] Mittal, A., Zisserman, A., \& Torr, P. "Hand detection using multiple proposals".In BMVC 2011 .

[12] Askar, S., Kondratyuk, Y., Elazouzi, K., Kauff, P., \& Schreer, O. "Vision-based skin-colour segmentation of moving hands for real-time applications." In IET Visual Media Production, 2004.(CVMP). 